\title{
Comparación de la mirmecofauna en un gradiente de reforestación
en bosques templados
del centro occidente de México
}

\section{Comparison of the ant's fauna in a reforestation gradient on temperate forests of Central Western Mexico}

\author{
Rafael Guzmán-Mendozal*, José Alejandro Zavala-Hurtado², Gabriela Castaño-Meneses ${ }^{3,5}$ \\ y Jorge Leonel León-Cortés ${ }^{4}$
}

\begin{abstract}
1 Departamento de Agronomía. División de Ciencias de la Vida. Campus Irapuato-Salamanca. Universidad de Guanajuato. Guanajuato México rgzmz@yahoo.com.mx

2 Departamento de Biología. Universidad Autónoma Metropolitana-Iztapalapa, México D.F.
\end{abstract}

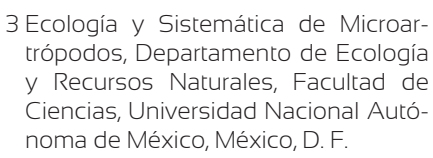

3 Ecología y Sistemática de Microartrópodos, Departamento de Ecología y Recursos Naturales, Facultad de Ciencias, Universidad Nacional Autónoma de México, México, D. F.

\author{
4 El Colegio de la Frontera Sur. Área \\ de Conservación de la Biodiversidad. \\ Departamento de Ecología y Siste- \\ mática Terrestre. San Cristóbal de las \\ Casas, Chiapas, México.
}

5 Unidad Multidisciplinaria de Docencia e Investigación, Facultad de Ciencias, Campus Juriquilla, Universidad Nacional Autónoma de México, Juriquilla, Querétaro, México.

\section{RESUMEN}

Se evaluó el cambio en la diversidad, riqueza, abundancia y similitud de las comunidades de hormigas en áreas con distinto impacto humano. Para ello se eligieron tres localidades con diferente tipo de vegetación ubicadas en el noroeste del Estado de México: árboles nativos, Quercus spp. (AN); combinación, Quercus spp. y elementos de reforestación (Cupressus lindleyi) (ZC); y reforestación, bosque modificado con C. lindleyi (R). Se colocaron 288 trampas de caída en febrero y marzo de 2009 en grupos de cuatro trampas en cuadrantes aleatorios de $10 \mathrm{~m}^{2}$. La composición y la abundancia de las comunidades variaron de acuerdo a las zonas de estudio. Los valores de riqueza y diversidad de especies fueron mayores en $\mathrm{R}$ en comparación con AN y ZC. Los resultados sugieren un efecto del cambio en la cobertura vegetal arbórea, sobre la estructura de las comunidades de hormigas. Especies como Temnothorax sp. 1 y Crematogaster sp., serían esperadas en zonas de bosque conservado, mientras que Pheidole sp. 1, Liometopum apiculatum y Camponotus atriceps, se consideran como especies relacionadas con la recolonización y la perturbación de un sitio.

PALABRAS ClAVE: Diversidad, perturbación, gremios tróficos, Formicidae.

\begin{abstract}
Changes in diversity, species richness, abundance and similarity patterns of ants' communities in different managed areas in Central Mexico were studied. Three areas located in the Northwest of the State of Mexico were selected: native trees area with Quercus spp. forest (AN), combination area with Quercus spp. forest and Cupressus lindleyi as reforestation species (ZC), and reforested area (R), an altered forest with C. lindleyi. A total of 288 pit-fall traps were placed from February to March 2009 in sets of four traps in random quadrants of $10 \mathrm{~m}^{2}$. The community composition and abundance shifted according to the site conditions. The values of richness and species diversity were higher in $\mathrm{R}$ in comparison with AN and ZC. The results suggest a relative effect of changes in floristic composition on ants' community. Species as Temnothorax sp. 1 and Crematogaster sp., were abundant in preserved forest areas. In contrast, Pheidole sp. 1, Liometopum apiculatum and Camponotus atriceps were relatively common in disturbed habitats.
\end{abstract}

KEYWORDS: Diversity, perturbation, trophic guilds, Formicidae. 


\section{INTRODUCCIÓN}

Los bosques templados de México son el segundo bioma más extenso del país (21\% del territorio) (Rzedowski, 1992) y uno de los más biodiversos a nivel mundial, debido, en parte, a que la mayoría de las especies que conforman el estrato arbóreo tienen su centro de origen en las montañas del territorio nacional (Fregoso et al., 2001; Valencia, 2004). De las aproximadamente 200 especies reconocidas del género Quercus en México, 70\% son endémicas (Zavala, 1998), mientras que del género Pinus hay presencia de poco más de 40 de las 100 especies reconocidas a nivel mundial, también con un alto grado de endemismos (Alba-López et al., 2003). A pesar de la gran riqueza florística reconocida, los bosques templados enfrentan un problema ambiental grave: desde el último cuarto del siglo $\mathrm{XX}$, la tasa de modificación de la cobertura vegetal se ha incrementado considerablemente (Medina y Tejero-Diez, 2006). En particular para este bioma, la tasa de deforestación de 1976 a 2000, fue estimada en 0,25\% anual, lo que representó una reducción en la cobertura vegetal original de un área de $20000 \mathrm{~km}^{2}$ (Mas et al., 2004).

La rápida modificación de los ecosistemas hace necesaria la identificación de organismos sensibles o indicadores, que proporcionen información rápida acerca del estado de conservación, alteración, o bien, el éxito en la restauración de un ambiente (Mattoni et al., 2000). En este ámbito, las hormigas son un grupo ecológicamente importante, tanto por su abundancia como por su diversidad, además de que desempeñan diferentes papeles ecológicos en los ecosistemas terrestres, como depredadoras, parásitas, herbívoras, detritívoras, granívoras, etc. (Hölldobler y Wilson, 1990), lo que las coloca en niveles tróficos altos, ya sea como especialistas o generalistas (Majer, 1983). Ocupan una posición clave dentro de los ecosistemas como ingenieros ecosistémicos, porque a partir de su actividad, pueden modificar la distribución, la abundancia y la dinámica de la comunidad vegetal (Whitford, 1978; Wilby y Shachak, 2000; ZavalaHurtado et al., 2000; Guzmán-Mendoza y Castaño-Meneses, 2007), cambiar las disposición de recursos y alterar las condiciones del suelo (Farji-Brener, 1992; Bestelmeyer y Wiens, 2001; Fortanelli y Servín, 2002). Lo anterior puede repercutir sobre propiedades del ecosistema, como los flujos de materia y energía (Naeem et al., 1999; Guzmán-Mendoza, 2004) y los procesos biogeoquímicos (Folgarait, 1998; Rojas, 2001). Además de tales atributos, se les captura con relativa facilidad, algunas especies son sensibles a las condiciones fluctuantes del ambiente (Philpott y Armbrecht, 2006). Por tal motivo, resultan ser útiles para el monitoreo de las condiciones ambientales (Shah et al., 2003; Stephens y Wagner, 2006; Gove et al., 2009, Philpott et al., 2010).

Se evaluó la hipótesis de que el cambio en la cobertura vegetal en el estrato arbóreo, como consecuencia de un gradiente de reforestación, puede ejercer efectos que se reflejan sobre los niveles tróficos superiores de las comunidades de hormigas. En este sentido, Luna-Cavazos et al. (2008) mostraron cómo el disturbio por aprovechamiento forestal, cambio en el uso de suelo, pastoreo e incendios, son determinantes de la comunidad herbácea. Si ello ocurre, los efectos del disturbio, en consecuencia, se pueden expresar también sobre las comunidades de insectos.

\section{OBJETIVOS}

Los objetivos del presente trabajo fueron evaluar los cambios en la diversidad, riqueza, abundancia y composición de la comunidad de hormigas, en función de las condiciones contrastantes en la conservación de la cobertura vegetal arbórea, así como proporcionar un listado con datos sobre los hábitos alimenticios de las especies de hormigas que componen la comunidad, en una zona de bosque templado del centro de México que no ha sido evaluada previamente y que, además, no cuenta con registros sobre la mirmecofauna de la región.

\section{MATERIALES Y MÉTODOS}

\section{Zonas de estudio}

Se seleccionaron tres sitios de estudio en la región noroeste del Estado de México, contrastantes en cuanto a la condición relativa del bosque. Las condiciones consideradas fueron: a) bosque con elementos arbóreos nativos (árboles nativos: AN; $19^{\circ} 45^{\prime} 48^{\prime \prime} \mathrm{N}, 9^{\circ} \quad 59^{\prime} \quad 20^{\prime \prime} \mathrm{O}, 2908$ 
msnm; clima templado semifrío, temperaturas entre $5^{\circ} \mathrm{C}$ y $12{ }^{\circ} \mathrm{C}$ y precipitación anual entre $200 \mathrm{~mm}$ y $1800 \mathrm{~mm}$ ), conformados por especies como Quercus rugosa Née, Q. laurina Humb. et Bonpl., Q. crassipes Humb. et Bonpl., Pinus sp., Arbutus sp. y Arbutus xalapensis Kunth, así como Alnus sp. y otras especies de bosque conservado, además de una mayor diversidad de formas de vida vegetal, de acuerdo con la clasificación de Flores y Álvarez-Sánchez (2004); b) bosque con una combinación de elementos arbóreos nativos (zona de combinación: ZC; $19^{\circ} 43^{\prime}$ 06” N, $100^{\circ} 05^{\prime} 38^{\prime \prime} \mathrm{O}, 2738 \mathrm{msnm}$; clima templado subhúmedo, temperaturas entre $18{ }^{\circ} \mathrm{C}$ y $20{ }^{\circ} \mathrm{C}$ y precipitación media anual entre $200 \mathrm{~mm}$ y $1800 \mathrm{~mm}$ ) con especies como $Q$. laurina, Q. rugosa, Q. crassipes, Q. sp., Pinus sp., Arbutus sp., y de reforestación como Cupressus lindleyi Klotzsch ex Endl.; c) bosque con elementos arbóreos de reforestación (R) con la especie C. lindleyi $\left(19^{\circ} 40^{\prime} 30^{\prime} \mathrm{N}, 100^{\circ} 05^{\prime} 51^{\prime \prime}\right.$ O, $2679 \mathrm{msnm}$; clima templado semifrío, temperaturas entre $5{ }^{\circ} \mathrm{C}$ y $12{ }^{\circ} \mathrm{C}$ y precipitación anual entre $200 \mathrm{~mm}$ y $1800 \mathrm{~mm}$ ); un análisis de diversidad de formas de vida mostró que fue el sitio con menor diversidad y riqueza de especies, en comparación de los otros dos, ya que presentó gran cantidad de herbáceas, y muy pocos arbustos y musgos (Guzmán-Mendoza, 2013).

\section{Recolecta de hormigas}

En cada sitio de estudio se eligieron 12 cuadrantes al azar de $10 \mathrm{~m}^{2}$ cada uno, con cuatro trampas de caída, distribuidas al azar al interior del cuadrante. Este esquema permitió colocar 48 trampas de caída por sitio durante febrero y 48 más en 12 cuadrantes, también por sitio, durante marzo de 2009, para un total de 288 trampas en todo el estudio. Estas trampas fueron elaboradas con envases de plástico de $500 \mathrm{ml}$ de capacidad, con $11 \mathrm{~cm}$ de diámetro y $13 \mathrm{~cm}$ de altura, a los que se les acondicionó una tapa que sostuvo un atrayente hecho a base de avena y miel. Las trampas fueron enterradas a nivel de suelo se mantuvieron cerradas por una semana, con el objeto de disminuir el efecto del disturbio. Una vez abiertas, los recipientes fueron llenados a la mitad únicamente con etilenglicol, sustancia no tóxica que evita la evaporación. Se añadieron unos granos de detergente, con la finalidad de disminuir la tensión superficial y facilitar el hundimiento de los insectos (Bestelmeyer et al., 2000), permanecieron abiertas durante 72 horas. Las hormigas capturadas fueron lavadas con agua corriente para quitar tierra y otros materiales, se almacenaron en viales con alcohol al $70 \%$, como conservador, para su posterior determinación en laboratorio. La determinación a nivel genérico se realizó utilizando las claves de Bolton (1994), así como las que se proporcionan en Mackay y Mackay (2005), para distintas especies. Una vez determinadas a nivel específico, las especies fueron agrupadas por hábitos alimenticios, de acuerdo con Brown (2000). El material se conserva en la Colección de Hormigas, del Laboratorio de Ecología y Sistemática de Microartrópodos, Facultad de Ciencias, UnAM.

\section{Análisis estadístico}

La abundancia de la fauna de hormigas por sitio se obtuvo a partir de la suma de individuos por especie por trampa. Para cada sitio se calculó la riqueza de especies (S), la diversidad con el índice de Shannon (H'), la dominancia con el índice de Simpson (1/D), y la equitatividad con el índice de Pielou (J'). Se aplicó un análisis de varianza de una vía con la prueba no paramétrica de Kruskal-Wallis, para comparar la variación en el número de individuos promedio capturados entre los sitios. En caso de diferencias significativas, se aplicó la prueba de LSD, diferencia mínima significativa, que se basa en la t de Student, con el objeto de reconocer los grupos que generan dichas diferencias. Con la finalidad de recalcular la probabilidad y tratar de evitar caer en el error tipo I, esta corrección consistió en dividir la probabilidad crítica entre el tamaño de muestra para cada comparación (Sokal y Rohlf, 1995). Además, se utilizó una prueba de $\chi^{2}$, mediante una tabla de contingencia $4 \times 3$ bidimensional, para evaluar si hubo o no diferencias significativas en la abundancia de los diferentes gremios alimenticios de las hormigas entre las zonas de estudio, dado que esta información puede servir para valorar el efecto de las hormigas sobre el desarrollo de la comunidad (Hendrix et al., 1988). En caso de valores significativos de $\chi^{2}$, se realizó un análisis de residuales estan- 
darizados, que permitió encontrar las categorías responsables de la desviación, aquellas que difieren del valor de la distribución normal estándar al 5\% de 1,96 (Everitt, 1977). Para esto se utilizó el programa SPSS ver 12 (INC. 2003 spsS For Windows Rel. 12.0 Chicago). Los índices de diversidad de Shannon fueron comparados mediante una prueba de $t$ modificada (Hutcheson, 1970), con el programa estadístico PAST (Hammer et al., 2001). Se calculó la riqueza estimada de especies mediante del estimador Chao 2, que es un modelo no paramétrico y que se basa en las abundancias de los individuos con datos de presencia ausencia (Krebs, 1999; Escalante, 2003). Los índices fueron calculados mediante el programa Biodiversity Pro ver. 2 (McAleece, 1997). Así mismo, para explorar los patrones de distribución de las especies de hormigas en las 96 muestras, se llevó a cabo un Análisis de Escalamiento Multidimensional No Métrico (NMDS, por sus siglas en inglés), sobre la matriz de similitud construida con el índice de Jaccard para generar un espacio de orde- nación de las muestras, donde se ajusta la compatibilidad de las disimilitudes en la matriz con la distancia euclidiana entre las muestras en el espacio de ordenación. Este método de ordenación ha probado ser adecuado para representar fielmente la similitud entre las muestras de comunidades en un espacio de ordenación (Minchin, 1987). Para este análisis se extrajeron dos dimensiones utilizando el programa XLSTAT 2011 (Addinsoft, 2011). Para todos los análisis realizados, fueron excluidas las trampas donde no se contabilizaron hormigas.

\section{RESULTADOS}

FAUNA MIRMECOLÓGICA POR SITIO DE ESTUDIO: Durante el periodo de trabajo de campo fueron recolectados 1517 individuos, pertenecientes a cinco subfamilias y 20 especies. La subfamilia que presentó la mayor abundancia fue Formicinae con 750 individuos, contabilizados en todas las trampas $(7,4 \pm 0,29$ por trampa), seguida de Myrmicinae con $383(3,8 \pm 0,11)$ y Dolichoderinae con 373 individuos $(5,4 \pm$

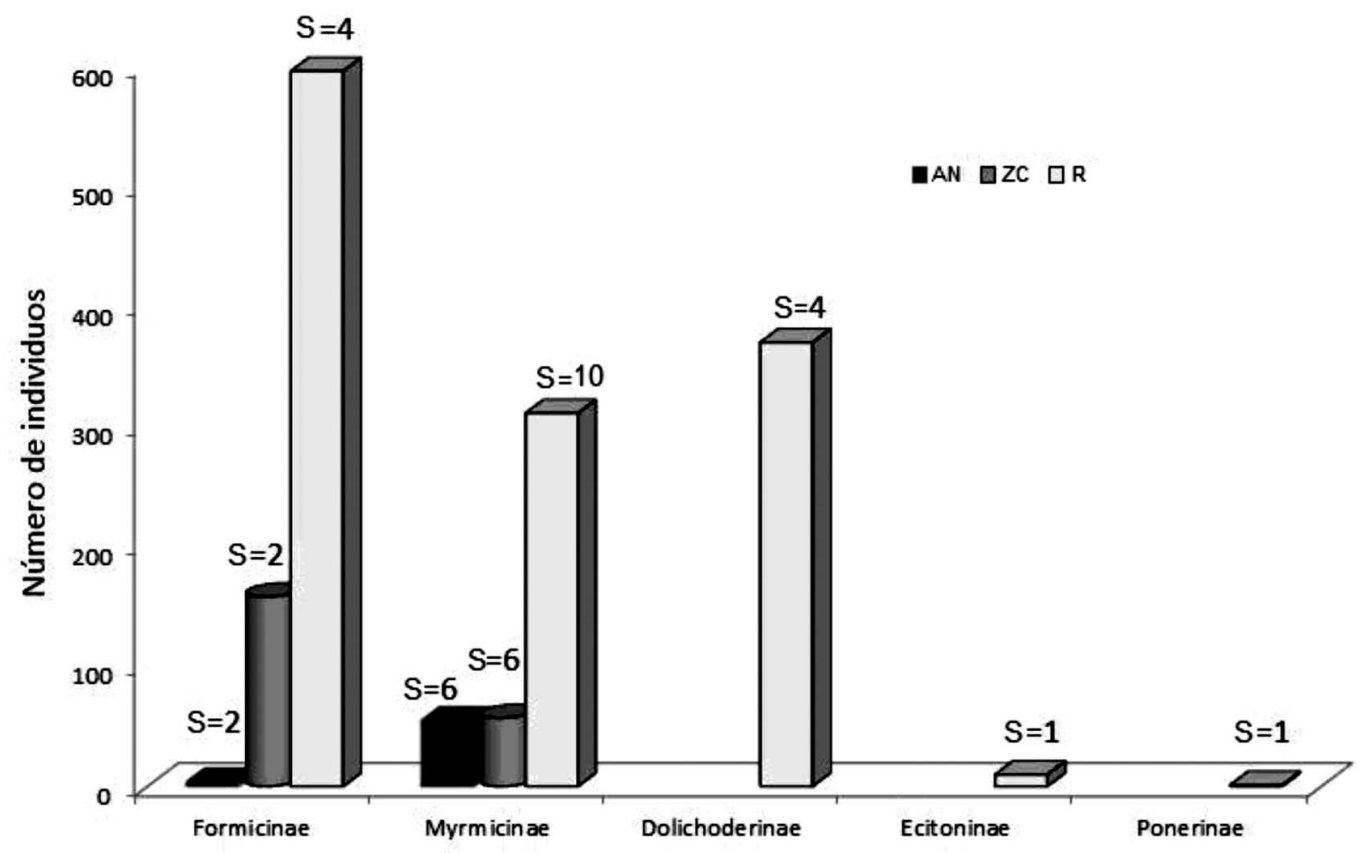

FIGURA 1. Cantidad de individuos por subfamilia y número de especies presentes en cada zona de estudio. Árboles nativos (AN); zona de combinación (ZN); reforestación (R), S = número de especies por familia dentro de cada sitio. 
0,40). El sitio con mayor número de organismos a este nivel taxonómico fue R, con cinco subfamilias, Formicinae y Myrmicinae se presentaron en los tres sitios de estudio, aunque en R se cuantificaron las abundancias más importantes. Especies de Ecitoninae y Ponerinae, sólo fueron recolectadas en $\mathrm{R}$, al igual que la mayoría de las especies de Dolichoderinae, a excepción de Dorymyrmex grandulus, que también se encontró en AN. Además, el mayor número de individuos y de especies por subfamilia $(S=20)$ registrado durante el estudio, fue encontrado en R (Fig. 1).

En términos generales, Myrmicinae, registró la mayor cantidad de especies $(S=10)$, siendo la más abundante Pheidole sp. 3, con 181 ejemplares. De Dolichoderinae, se encontraron cuatro especies, con Liometopum apiculatum (Mayr), como la más abundante por el número de individuos contabilizados. La subfamilia Formicinae registró cuatro especies, con Camponotus sp.2, la más importante en términos de su abundancia (615 individuos; 17,5 \pm 0,67 por trampa en todos los sitios). En Ecitoninae y Ponerinae se identificó sólo a una especie, Labidus coecus (Latreille) y Odontomachus clarus (Roger), respectivamente. Las especies, Dorymyrmex bureni (Trager), Camponotus sp.1, Formica sp. y O. clarus, obtuvieron únicamente un registro durante todo el estudio (Tabla 1).

Por otro lado, a excepción de D. grandulus, el resto de especies de Dolichoderinae se presentaron solamente en R. Además, la mayor parte de las especies encontradas fueron más abundantes en esta zona, por ejemplo, L. apiculatum, Camponotus sp. 2 y Pheidole sp. 3. En contraste, pocas especies fueron abundantes en AN (Pheidole sp. 2 y Temnothorax sp. 1) y ZC, (Camponotus sp. 2) (Fig. 2). Finalmente, el resultado de la prueba de Kruskal-Wallis

TABLA 1. Especies colectadas, gremios alimenticios y promedio de individuos por trampa, presentes en los sitios de estudio.

\begin{tabular}{|c|c|c|c|c|c|}
\hline \multirow{2}{*}{ Subfamilia/especie } & \multirow{2}{*}{ Hábitos alimenticios } & \multirow{2}{*}{ Número de individuos promedio $\pm S E$} & \multicolumn{3}{|c|}{ Presencia } \\
\hline & & & AN & ZC & $\mathrm{R}$ \\
\hline \multicolumn{6}{|l|}{ Dolichoderinae } \\
\hline Dorymyrmex grandulus & $\mathrm{D}, \mathrm{HD}, \mathrm{CR}, \mathrm{NC}$ & $0,12 \pm 0,2$ & $x$ & & $x$ \\
\hline Dorymyrmex flavopectus & $\mathrm{HD}$ & $0,07 \pm 0,1$ & & & $x$ \\
\hline Dorymyrmex bureni & $\mathrm{D}$ & $0,01 \pm 0,1$ & & & $x$ \\
\hline Liometopum apiculatum & $\mathrm{HD}$ & $6,4 \pm 0,8$ & & & $x$ \\
\hline \multicolumn{6}{|l|}{ Ecitoninae } \\
\hline Labidus coecus & $\mathrm{D}, \mathrm{CR}$ & $0,1 \pm 0,3$ & & & $x$ \\
\hline \multicolumn{6}{|l|}{ Formicinae } \\
\hline Camponotus atriceps & $\mathrm{CR}$ & $2,3 \pm 0,8$ & & $x$ & $x$ \\
\hline Camponotus sp.1 & $\mathrm{HD}, \mathrm{D}, \mathrm{CR}$ & $0,01 \pm 0,1$ & & & $x$ \\
\hline Camponotus sp.2 & $\mathrm{HD}, \mathrm{D}, \mathrm{CR}$ & $10,9 \pm 0,6$ & $\mathrm{x}$ & $x$ & $\mathrm{x}$ \\
\hline Formica sp. & $\mathrm{HD}, \mathrm{CR}$ & $0,01 \pm 0,1$ & & & $x$ \\
\hline \multicolumn{6}{|l|}{ Myrmicinae } \\
\hline Crematogaster sp. & $\mathrm{D}, \mathrm{HD}, \mathrm{CR}$ & $0,1 \pm 0,2$ & $x$ & & $x$ \\
\hline Monomorium cyaneum & $\mathrm{D}, \mathrm{HD}, \mathrm{GR}$ & $0,5 \pm 0,5$ & $x$ & & $x$ \\
\hline Pheidole sp.1 & $G R, C R, D, H D$ & $0,4 \pm 0,4$ & & $x$ & $x$ \\
\hline Pheidole sp.2 & $G R, C R, D, H D$ & $1,2 \pm 0,2$ & $x$ & $x$ & $x$ \\
\hline Pheidole sp.3 & $G R, C R, D, H D$ & $32 \pm 0,5$ & $x$ & $x$ & $x$ \\
\hline Pheidole sp.4 & $G R, C R, D, H D$ & $0,03 \pm 0,1$ & & $x$ & $x$ \\
\hline Pheidole sp.5 & $G R, C R, D, H D$ & $0,03 \pm 0,1$ & & & $x$ \\
\hline Temnothorax sp.1 & $\mathrm{CR}$ & $0,5 \pm 0,3$ & $x$ & $x$ & $x$ \\
\hline Temnothorax sp.2 & $\mathrm{CR}$ & $0,5 \pm 0,3$ & & & $x$ \\
\hline Tetramorium sp. & $\mathrm{HD}, \mathrm{CR}$ & $0,1 \pm 0,1$ & $x$ & $x$ & $x$ \\
\hline \multicolumn{6}{|l|}{ Ponerinae } \\
\hline Odontomachus clarus & $\mathrm{D}$ & $0,01 \pm 0,1$ & & & $x$ \\
\hline
\end{tabular}

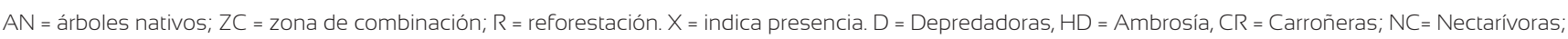
$\mathrm{GR}=$ Granívoras 

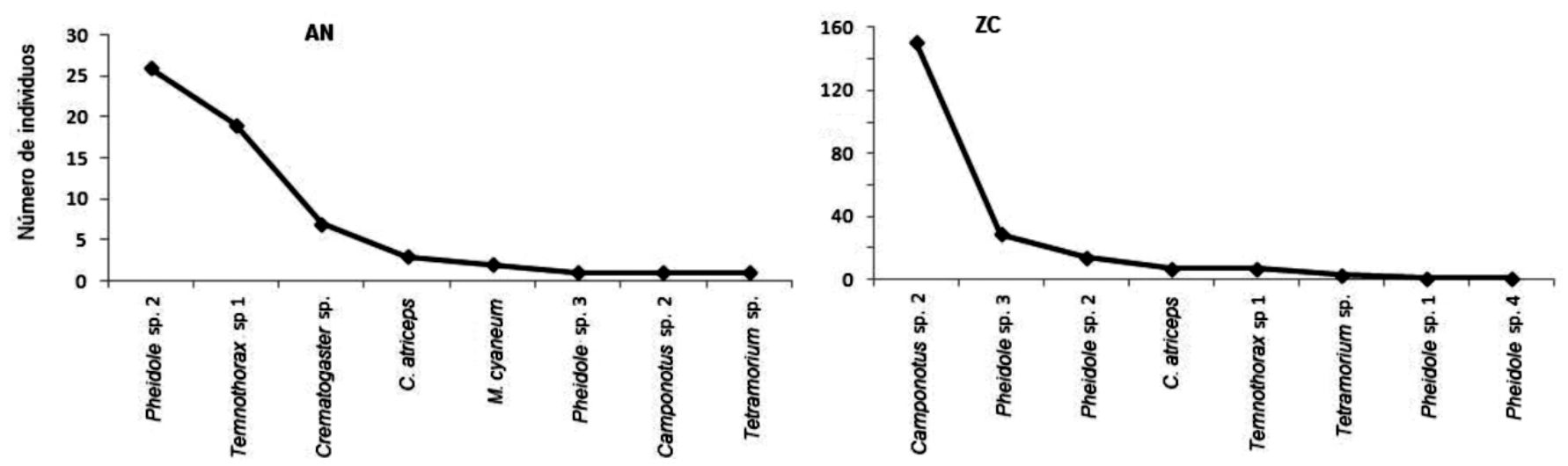

Especies en orden de abundancia

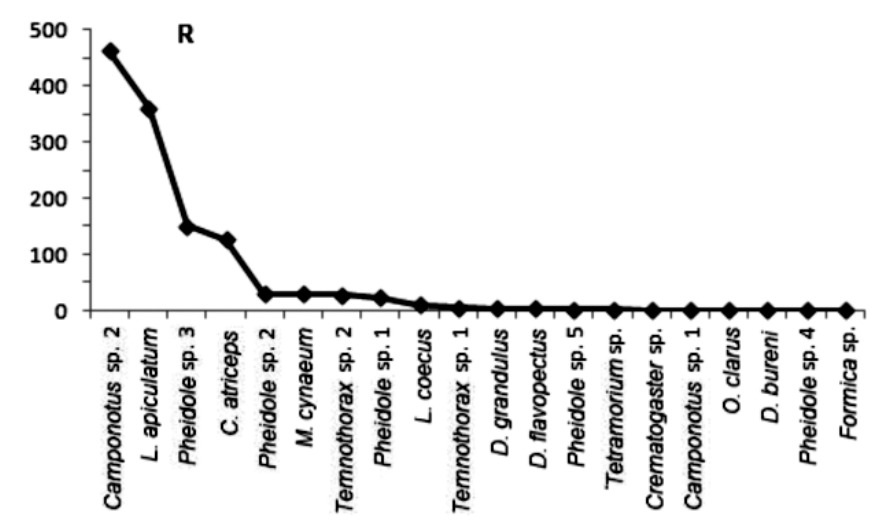

FIGURA 2. Abundancia de especies de hormigas, presentes en cada zona de estudio. AN= árboles nativos; ZN = zona de combinación; $\mathrm{R}=$ reforestación.

$(\mathrm{K}-\mathrm{W})$, indicó que el número promedio de individuos capturados durante el periodo de estudio, fue estadísticamente diferente entre las zonas $\left(\mathrm{K}_{-} \mathrm{W}_{0,05 ; 2}=18,002\right.$; $\mathrm{p}<0,0001)$, presentando $\mathrm{R}$ un promedio mayor de hormigas capturadas $(67,7 \pm 3,6)$, en comparación con AN $(3,1 \pm$ $0,9)$ y ZC $(11,3 \pm 2,3)$, que también registraron diferencias en intervalo de la cantidad de organismos atrapados. El análisis de LSD mostró diferencias significativas en todos los caos (AN-ZC = 8,21; $\mathrm{p}=0,035$. AN-R = 64,68; $\mathrm{p}=0,00075 . \mathrm{ZC}-\mathrm{R}=56,47 ; \mathrm{p}=0,0016)$.

COMUNIDAD MIRMECOLÓGICA: El resultado del análisis comparativo, sugiere diferencias significativas entre los índices de diversidad en cada zona. En este sentido, la diversidad de AN (árboles nativos), fue mayor a ZC (zona de combinación) $\left(\mathrm{t}_{0,05(2) 112,5}=2,53 ; \mathrm{p}=0,012\right)$, pero menor a la diversidad encontrada en $\mathrm{R}$ (reforestación) $\left(\mathrm{t}_{0,05(2) 65,7}=\right.$ $2,45 ; \mathrm{p}=0,016)$. La comparación entre ZC y R, indica que el primer sitio, fue el de menor diversidad de todos demás $\left(\mathrm{t}_{0,05(2) 275,7}=8.08 ; \mathrm{p}<0,0001\right)$ (Tabla 2). En contraste, $\mathrm{R}$ no sólo fue el sitio de mayor diversidad, sino también el de mayor riqueza de especies $(S=20)$.

Por otro lado, de acuerdo con el índice de Simpson (Tabla 2), la dominancia observada en AN está determinada fundamentalmente por la abundancia de dos espe- 
cies, que en orden de importancia (abundancia), fueron las mirmecinas: Pheidole sp.2 y Temnothorax sp. 1 (Fig. 2). En ZC, la especie más importante para la comunidad por su abundancia es Camponotus sp.2 (Fig. 2). Finalmente, en R, las especies que estructuraron a la comunidad en términos de su dominancia, fueron Camponotus sp. 2 y L. apiculatum (Fig. 2). Los resultados muestran una marcada heterogeneidad en $\mathrm{AN}$, a pesar de tener una diversidad menor que R. En este sentido, el índice de equitatividad (J') sugiere una mayor dominancia en $\mathrm{R} y$ AN (Tabla 2)

Todas las especies registradas en el estudio se presentaron en $\mathrm{R}$ y algunas fueron observadas únicamente en este sitio. Por ejemplo, para la familia Dolichoderinae, las especies D. flavopectus, D. bureni y L. apiculatum; la única especie de Ecitoninae: L. coecus; dos especies de Formicinae: Camponotus sp. 1 y Formica sp.; Temnothorax sp. 2 (Myrmicinae) y O. clarus (Ponerinae). En cuanto a AN y ZC, todas las especies encontradas en estos sitios fueron compartidas, ya sea entre ellas o con R (Fig. 2, Tabla 1).

El análisis de $\chi^{2}$ muestra diferencias significativas en la distribución de los gremios alimenticios entre los tres ambientes estudiados $\left(\chi_{0,05 ; 6}^{2}=40,75 ; \mathrm{p}<0,0001\right)$. En este sentido, la presencia de los gremios, puede estar relacionada con las características de las zonas, en cuanto a los

TABla 2. Valores para: riqueza de especies (S), índices de diversidad de Shannon (H') y Simpson (1/D), diversidad máxima de especies (H'max) e índice de equitatividad (J') para las comunidades de hormigas por sitio de colecta.

\begin{tabular}{cccc}
\hline Parámetro & AN & $Z C$ & $R$ \\
\hline $\mathrm{S}$ & 8 & 8 & 20 \\
\hline $\mathrm{H}^{\prime}$ & 0,62 & 0,44 & 0,74 \\
\hline $1 / \mathrm{D}$ & 3,4 & 1,9 & 4,1 \\
\hline $\mathrm{H}^{\prime}$ max & 0,90 & 0,90 & 1,30 \\
\hline$J^{\prime}$ & 0,69 & 0,49 & 0,56 \\
\hline
\end{tabular}

AN = árboles nativos, ZC = combinación, $\mathrm{R}=$ reforestación recursos que pueden explotar en cada una. Comparando los valores absolutos encontrados a partir del resultado de residuales estandarizados, con $5 \%$ de la desviación normal estándar $(1,96)$, es posible observar que muchos de los residuales ajustados son significativos, principalmente para las zonas ZC y AN. En cuanto a R, sólo HD fue diferente de la desviación normal estándar, además de que en esta zona se encontró Dorymyrmex grandulus, considerada también, nectarívora (NC) y que fue la única especie con este tipo de actividad alimenticia (Tabla 3).

Las curvas de acumulación de especies, sugieren valores similares para ZC y AN, que fueron más bajos en comparación con R. Con un mayor esfuerzo de muestreo, el resultado indica que probablemente se podrían incrementar o igualar los valores observados en ZC y AN, pero no llegar a la riqueza potencial estimada para $R$ (Fig. 3).

EFECTO DE LAS MODIFICACIONES DEL HÁbITAT. El análisis de ordenación mostró que las comunidades se ordenan a lo largo de un gradiente que va de la zona más conservada (AN), pasando por la zona con elementos de la vegetación original y de reforestación (ZC), hasta las muestras de la zona sujeta a la mayor modificación de la comunidad vegetal (R). Este gradiente está definido por los dos primeros ejes del NMDS, con un estrés de 0,193, que se considera como una solución aceptable para la representa-

TABLA 3. Valores de los residuales ajustados mediante el método de residuales estandarizados, comparados con el valor de la desviación normal estándar al 0,05 de 1,96 para las comunidades de hormigas por sitio de colecta y gremio trófico.

\begin{tabular}{ccccc}
\hline Zonas & \multicolumn{4}{c}{ Gremios } \\
\cline { 2 - 5 } & $D$ & $H D$ & $C R$ & $G R$ \\
\hline AN & $-7,4$ & $-20,9$ & 12,4 & 55,2 \\
\hline ZC & 10,8 & $-7,8$ & 5,8 & $-17,9$ \\
\hline R & $-1,9$ & 2,7 & $-1,9$ & 0,9 \\
\hline
\end{tabular}

AN = árboles nativos; $Z C$ = zona de combinación; $R$ = reforestación. $D$ = Depredadoras, $\mathrm{HD}=$ Ambrosía, $\mathrm{CR}=$ Carroñeras; $\mathrm{GR}=\mathrm{G}$ ranívoras. 


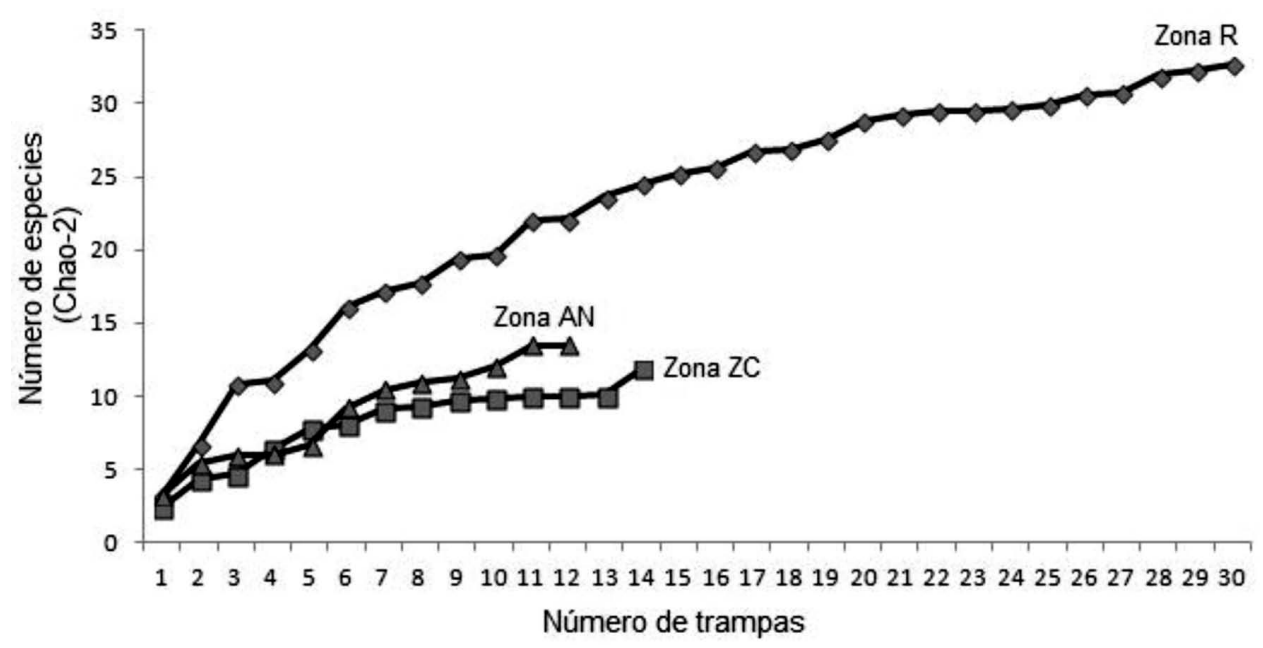

FIgURA 3. Curvas de estimación potencial de la riqueza de especies de hormigas para cada zona de estudio. $\mathrm{AN}$ = árboles nativos; $\mathrm{ZN}$ = zona de combinación; $\mathrm{R}$ = reforestación

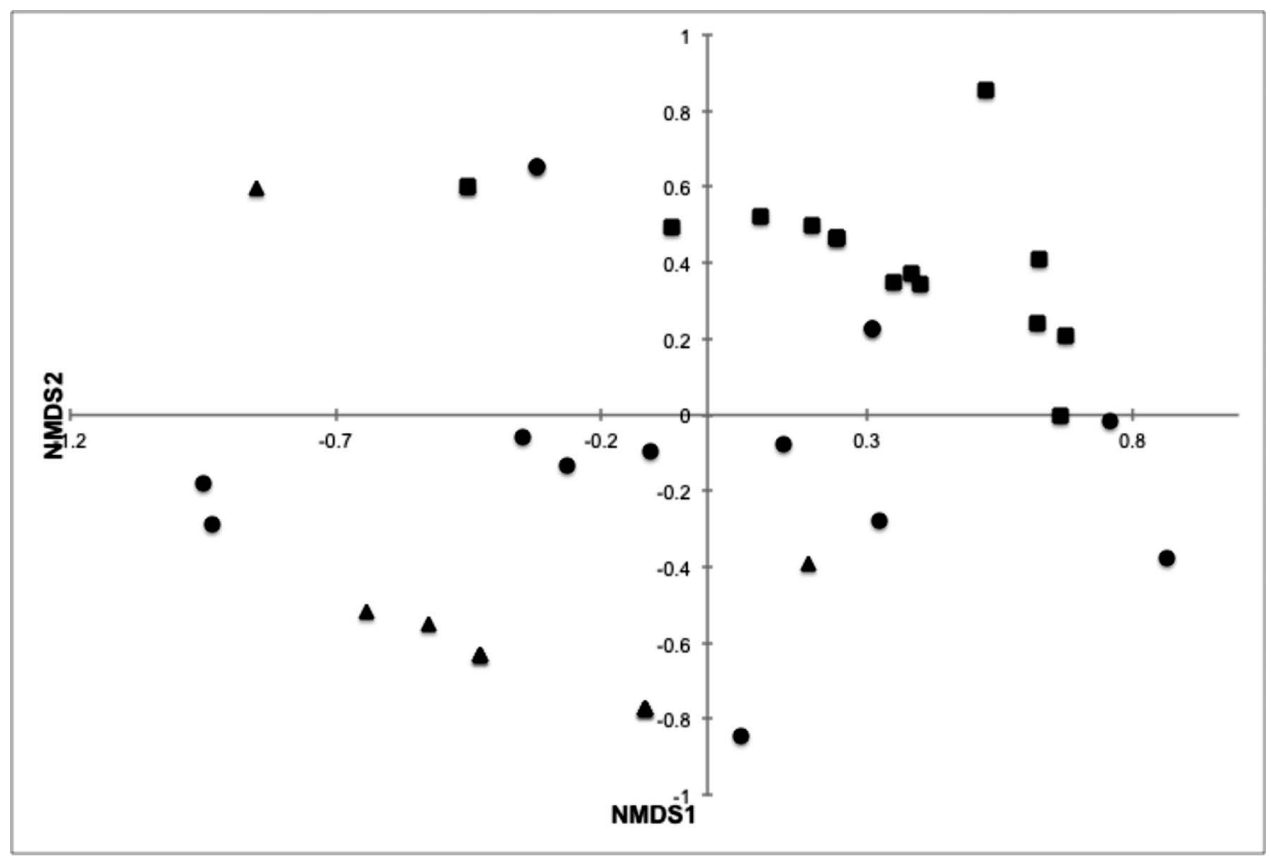

Figura 4. Diagrama de dispersión en dos ejes del Análisis de Escalamiento Multidimensional No Métrico (NMDS) de las comunidades de hormigas en zonas con diferente condición de la cobertura vegetal arbórea. $\mathrm{AN}=$ árboles nativos $(\boldsymbol{\Lambda}) ; \mathrm{ZN}=$ zona de combinación $(\bullet)$; $\mathrm{R}=$ reforestación $(\mathbf{-})$. 
ción de las similitudes entre las muestras en el espacio de ordenación (Kruskal, 1964) (Fig. 4). De acuerdo con lo anterior, especies como Temnothorax sp. 1 y Crematogaster sp., corresponden a la zona conservada, mientras que Pheidole sp. 3, Pheidole sp. 2, Tetramorium sp. y Pheidole sp .4, aparecen relacionadas con ZC. Especies como Pheidole sp. 1, L. apiculatum y C. atriceps, parecen tener una preferencia mayor por R. También se puede apreciar una segregación clara de las muestras de acuerdo con el sitio al que pertenecen, aunque la heterogeneidad en la composición de especies dentro de cada zona es relativamente alta. Así, las muestras para AN tuvieron una similitud promedio de 47,36 $\pm 26,02 \%$. En ZC el valor promedio de semejanza entre las muestras fue de $26,55 \pm 29,87 \%$ y, en $R$, el valor promedio fue de 42,85 $\pm 35,93 \%$. Los resultados obtenidos muestran que la fauna de ZC fue más parecida a AN que a R. Especies como C. atriceps, Pheidole sp. 4 y Tetramorium sp. fueron elementos compartidos entre ZC y AN. Camponotus sp. 1, Pheidole sp. 4 y Pheidole sp. 1. se presentaron tanto en $\mathrm{ZC}$ como en $\mathrm{R}$.

\section{DISCUSIÓN}

Los resultados sugieren que las comunidades de hormigas se han visto afectadas por el cambio en la cobertura vegetal arbórea, originada por el gradiente de reforestación. Se observa un gradiente entre las zonas AN (la más conservada) hasta $\mathrm{R}$, que corresponde a la zona con mayor modificación ambiental por la reforestación, donde ZC es el lugar intermedio en cuanto a conservación. El hecho de que $\mathrm{R}$ sea la zona con mayor diversidad, puede deberse a que las hormigas son colonizadoras exitosas en sitios con perturbaciones frecuentes (Folgarait, 1998), siendo Pheidole sp.1, L. apiculatum y C. atriceps, especies indicadoras tanto de un proceso de recolonización progresivo, como de la perturbación de un sitio, lo que se ha observado para algunas especies de Dolichoderinae que son funcionalmente un grupo dominante de acuerdo con Andersen (2000) y Andersen y Majer (2004). Estudios realizados en bosques perturbados de eucaliptos en Australia, en zonas perturbadas del Chaco Argentina y en bosques templados en Georgia, muestran que algunas especies de Dolichoderinae, pertenecientes a los géneros Dorymyrmex y Forelius, son comunes en hábitats alterados (Graham et al., 2004). Sin embargo, a diferencia de estos estudios, los resultados obtenidos muestran que el disturbio ocurrido en las zonas de trabajo incrementa además de la abundancia, la diversidad de hormigas, aunque con una menor equitatividad. Lo anterior puede deberse a factores intrínsecos del disturbio, como la frecuencia, la intensidad y la fuente de la perturbación, lo que a su vez afecta los ensambles de la comunidad (Fukami y Morin, 2003). Por ejemplo, se ha observado que las hormigas arbóreas son más sensibles a la fragmentación que las hormigas terrestres (Gove et al., 2009); mientras que el estrés hídrico en las plantas promueve respuestas tanto negativas como positivas y neutras, sobre la abundancia y la diversidad que depende de los gremios estudiados (Huberty y Denno, 2004). Andersen (1991a) encontró que especies de hormigas crípticas y generalistas eran comunes en zonas sin disturbio, mientras que las hormigas oportunistas y especializadas lo eran en zonas con disturbio por fuego. En el presente trabajo, la zona R registró una mayor abundancia de hormigas con hábitos alimenticios carroñeros y de ambrosía.

Las especies que actúan como carroñeras, bien pueden estar funcionando como las oportunistas observadas por Andersen (1991a), la ambrosía, por su parte, puede estar favorecida por la presencia de hormigas del género Camponotus, específicamente del morfo sp. 2, que pueden estar asociadas a otros organismos como hemípteros de la familia Membrasidae, que estuvieron presentes en la zona de estudio (Guzmán-Mendoza, 2013) y que se ha publicado, que son atendidos por hormigas (Schuh y Slater, 1995). Sin embargo, inferir el efecto de las modificaciones del ambiente sobre la comunidad de hormigas basado en los hábitos alimenticios debe tomarse con cautela debido a que para algunas especies no se conoce totalmente la dieta, como en el caso de Temnothorax (Rueppell y Kirkman, 2005).

Una explicación alterna refiere a que tales asociaciones, pueden corresponder a los grupos funcionales propuestos por Andersen (1991b). En este sentido, las 
Dolichoderinae y las generalistas Pheidole, funcionalmente definidas como dominantes, fueron abundantes en $\mathrm{R}$, así como las subordinadas Camponotus.

El gradiente de conservación sugerido por los resultados donde AN y R son los extremos de un bosque conservado a uno completamente modificado, muestra que éstos tienen una diversidad mayor que el punto intermedio. Lo anterior parece contraponerse a lo predicho por la teoría del disturbio intermedio y a lo observado en otros grupos donde la riqueza aumenta hacia los sitios con disturbio intermedio (Premauer y Vargas, 2004). Una razón puede radicar en la naturaleza de los factores involucrados en el disturbio (Guzmán-Mendoza et al., 2010) y que pueden condicionar la identidad de las especies que conforman una comunidad. En particular en R, ocurren incendios accidentales durante la temporada seca, mientras que en ZC, el disturbio es ocasionado por las veredas que marcan los habitantes, los animales de pastoreo y la extracción de leña, lo que no se presenta en AN.

Por otro lado, la baja abundancia de hormigas en AN, puede responder al hecho de que este atributo de la comunidad, decrece al incrementarse la madurez del bosque y, por lo tanto, las condiciones conservadas del mismo (Sonthichai et al., 2006); pero además, los resultados son consistentes con la hipótesis de la limitación termal: temperaturas frías limitan la capacidad de las hormigas para obtener recursos (Kaspari et al., 2000) y, dado que en esta zona se registran las temperaturas más bajas, resulta una posible explicación. De acuerdo con los resultados, especies como Temnothorax sp. 1 y Crematogaster sp., serían especies esperadas en zonas de bosque conservado. La baja diversidad encontrada en AN puede indicar que las hormigas se encuentren también en otros ambientes dentro de la zona; las epífitas como bromelias, helechos y musgos, que crecen sobre las ramas de los encinos, y que son más abundantes en esta zona, y prácticamente inexistentes en $\mathrm{R}$, pueden estar incrementando la heterogeneidad ambiental y por lo tanto, generar hábitats a especies que no visitan el suelo o al menos que lo hacen pocas veces, como Crematogaster sp., que se encontró en AN. Lo anterior puede explicar el hecho de que AN fue una zona con menor diversidad que R, no obstante que la heterogeneidad de la comunidad fue mayor a cualquiera de las otras dos zonas. Además, existen antecedentes donde se ha mostrado una relación positiva, entre la heterogeneidad ambiental proporcionada por la diversidad vegetal y la diversidad de artrópodos (Siemann et al., 1998).

A pesar de que se observa un incremento de la diversidad de hormigas en la zona con un cambio drástico en la cobertura vegetal, la modificación a través de reforestaciones monoespecíficas puede homogeneizar la diversidad de insectos debido a que disminuye la hetereogeneidad espacial, lo cual puede repercutir sobre procesos importantes de los ecosistemas como el flujo de nutrientes, la composición de la comunidad de productores y sobre la productividad primaria del ambiente (Guzmán-Mendoza, 2004).

\section{CONCLUSIONES}

De acuerdo con los resultados, se observa un efecto, dependiendo del grado de conservación, de las condiciones de los bosques sobre la riqueza, diversidad, composición y estructura de las comunidades de hormigas presentes en los sitios de colecta. En este sentido, la zona $\mathrm{R}$ muestra valores altos en estos parámetros de la comunidad, seguido de AN y finalmente de ZC, la zona intermedia. En principio esto sugiere que la hipótesis del disturbio intermedio no se cumple con los datos encontrados, mientras que otras hipótesis, como la limitación termal (Klok et al., 2004), sí se sustenta con los resultados obtenidos. Para corroborar dichos resultados, es necesario incrementar los puntos de muestreo, aunque se debe considerar que las condiciones sean lo más parecidas entre sí.

Se propone que la identidad de las especies de hormigas presentes en la zona AN, puede ser utilizada para apoyar una estrategia adecuada de conservación, manejo o reforestación de los bosques de la región, como lo muestra la presencia de especies como Crematogaster sp. No obstante, para reforzar estos resultados se deben tomar en cuenta aspectos como la heterogeneidad espacial a la que responden sensiblemente las hormigas, al modificar las 
abundancias de sus poblaciones y la composición de sus comunidades.

\section{AGRADECIMIENTOS}

Los autores agradecen a Susana Valencia Ávalos (Facultad de Ciencias de la UNAM) y a Carmen De la Paz PérezOlvera (UAM-I), por la identificación de las especies arbóreas presentes en la zona de estudio. A las autoridades locales: Pascasio Ramírez Ramírez; Rosendo Santana Martínez y Javier Téllez, por las facilidades y apoyo en el trabajo de campo. A Josefina Calzontzi Marín y Teresa Colín Calzonzi por su ayuda en la separación de insectos. Al Conacyt por la beca de posgrado y al programa del Doctorado en Ciencias Biológicas de la UAM al que estuvo adscrito el primer autor. Finalmente a los dos revisores anónimos, con cuyas sugerencias se mejoró significativamente el escrito.

\section{REFERENCIAS}

Addinsoft. 2011. XLSTAT 2011. Addinsoft. Paris. 230 p.

Alba-López, M.P., M. González-Espinosa, N. Ramírez-Marcial y M.A. Castillo-Santiago. 2003. Determinantes de la distribución de Pinus spp. en la altiplanicie central de Chiapas, México. Boletín de la Sociedad Botánica de México 73:7-15.

Andersen, A.N. 1991a. Responses of ground foraging ant communities to three experimental fire regimes in a savanna forest of tropical Australia. Biotropica 23(4b):575-585.

Andersen, A.N. 1991b. Parallels between ants and plants: implications for community ecology. In: C. Huxley y D.F. Cutler, eds. Ant-plant interactions. Oxford Science Publications. p:539-558.

Andersen, A.N. 2000. A global ecology of rain forest ants: functional groups in relation to stress and disturbance. In: D. Agosti, J.D. Majer, L.E. Alonso y T.R. Schultz, eds. Ants: standard methods for measuring and monitoring biodiversity. Smithsonian Institution Press. Washington, D.C. p:25-34.

Andersen, A.N. y J.D. Majer. 2004. Ants show the way down under: invertebrates as bioindicators in land management. Frontiers in Ecology Environment 2(6):291-298.
Bestelmeyer, B.T., D. Agosti, L.E. Alonso, C.R.F. Brandao, W.L. Brown Jr., J.H.C. Delabie y R. Silvestre. 2000. Field techniques for study of ground-dwelling ants: an overview, description and evaluation. In: D. Agosti, J.D. Majer, L.E. Alonso y T.R. Schultz, eds. Ants: standard methods for measuring and monitoring biodiversity. Smithsonian Institution Press. Washington, D.C. p:122-144.

Bestelmeyer B.T. y J.A. Wiens. 2001. Ant biodiversity in semiarid landscape mosaics: The consequences of grazing vs. natural heterogeneity. Ecological Applications 11(4): 1123-1140.

Bolton, B. 1994. Identification guide to the ant genera of the World. London, Harvard University Press. 222 p.

Brown, W.L. 2000. Diversity of ants. In: D. Agosti, J.D. Majer, L.E. Alonso y T.R. Schultz, eds. Ants: standard methods for measuring and monitoring biodiversity. Smithsonian Institution Press. Washington, D.C. p:45-79.

Escalante, E.T. 2003. ¿Cuántas especies hay? Los estimadores no paramétricos de Chao. Elementos 52:53-56.

Everitt, B.S. 1977. The analysis of contingency tables. Chapman and Hall/CRC. Londres. 128 p.

Farji-Brener, A.G. 1992. Modificaciones al suelo realizadas por hormigas cortadoras de hojas (Formicidae: Attini): una revisión de sus efectos sobre la vegetación. Ecología Austral 2:87-94.

Flores, J.S. y J. Álvarez-Sánchez. 2004. Flora y vegetación. In: F. Bautista S., H. Delfín G., J.L. Palacio P., M del C. Delgado, eds. Técnicas de muestreo para manejadores de recursos naturales. México. UNAM, UAY, Conacyt, INE. p:303-327.

Folgarait, P. 1998. Ant biodiversity and its relationship to ecosystem functioning: A review. Biodiversity and Conservation 7(9):1221-1244.

Fortanelli, M.J. y M.E. Servín. 2002. Desechos de hormiga arriera (Atta mexicana Smith), un abono orgánico para la producción hortícola. Terra 20 (2):153-160.

Fregoso, A., A. Velázquez, G. Bocco y G. Cortéz. 2001. El enfoque de paisaje en el manejo forestal de la comunidad indígena de Nuevo San Juan Parangaricutiro, Michoacán, México. Investigaciones Geográficas, Boletín del Instituto de Geografía. UNAM. 46:58-77. 
Fukami, T. y P.J. Morin. 2003. Productivity-biodiversity relationships depend on the history of community assembly. Nature 424(6965):423-426.

Gove, A.D., J.D. Majer y V. Rico-Gray. 2009. Ant assemblages in isolated trees are more sensitive to species loss and replacement than their woodland counterparts. Basic and Applied Ecology 10(2):187-195.

Graham, J.H., H.H. Hughie, S. Jones, K. Wrinn, A.J. Krzysik, J.J. Duda, D.C. Freeman, J.M. Emlen, J.C. Zak, D.A. Kovacic, C. Chamberlin-Graham y H. Balbach. 2004. Habitat disturbance and the diversity and abundance of ants (Formicidae) in the Southeastern Fall-Line Sandhills. Journal of Insect Science 4(30):1-15. En línea: insectscience.org/4.30.

Guzmán-Mendoza, R. 2004. Patrones de actividad de forrajeo de Pogonomyrmex barbatus en el valle semiárido de Zapotitlán Salinas, Puebla. Tesis de Maestría. Universidad Autónoma Metropolitana. 116 p.

Guzmán-Mendoza, R. y G. Castaño-Meneses. 2007. Selected foraging activity of Camponotus rubrithorax Forel (Hymenoptera: Formicidae) in the Zapotitlán Salinas valley, Puebla. Sociobiology 50(2):435-448.

Guzmán-Mendoza, R., G. Castaño-Meneses y M.C. HerreraFuentes. 2010. Variación espacial y temporal de la diversidad de hormigas en el Jardín Botánico del valle de Zapotitlán de las Salinas. Revista Mexicana de Biodiversidad 80(2):427-435.

Guzmán-Mendoza, R. 2013. Efectos de la reforestación sobre los patrones de diversidad de artrópodos en una región de bosque templado del Centro de México. Tesis de Doctorado. Universidad Autónoma Metropolitana. 283 p.

Hammer, Ø., D.A.T. Harper y P.D. Ryan, 2001. PAST: Paleontological statistics software package for education and data analysis. Palaeontologia Electronica 4(1):9pp. http:// palaeo-electronica.org/2001_1/past/issue1_01.htm; última consulta: 16. II.2011.

Hendrix, S.D., V.K. Brown y H. Dingle. 1988. Arthropod guild structure during early old field succession in a new and old world site. Journal of Animal Ecology 57(3):1053-1065.

Hölldobler, B. y E.O. Wilson. 1990. The Ants. Harvard University Press. Cambridge, Massachusetts. 732 p.
Hutcheson, K. 1970. A test for comparing diversities based on the Shannon formula. Journal of Theoretical Biology 29(1):151-154.

Huberty, A.F. y R.F. Denno. 2004. Plant water stress and its consequences for herbivorous insects: a new synthesis. Ecology 85(5):1383-1398.

Kaspari, M., L. Alonso y S. O’Donnell. 2000. Three energy variables predict ant abundance at a geographical scale. Proceedings of the Royal Society of London $B$ 267(1442):485-489.

Klok, C.J., B.J. Sinclair y S.L. Chown. 2004. Upper thermal tolerance and oxygen limitation in terrestrial arthropods. The Journal of Experimental Biology 207:23612370.

Krebs, C.J. 1999. Ecological methodology. 2a ed. Benjamin Cummings. Menlo Park California. 620 p.

Kruskal, J.B. 1964. Nonmetric Multidimensional Scaling: a numerical method. Psychometrika 29(2): 115-129

Luna-Cavazos, M., A. Romero-Manzanares y E. García-Moya. 2008. Afinidades en la flora genérica de piñonares del norte y centro de México: un análisis fenético. Revista Mexicana de Biodiversidad 79(2):449-458.

McAleece, N. 1997. Biodiversity Professional Beta. Version 2.0.The Natural History Museum and The Scottish Association for Marine Science.

Mackay, W. y E. Mackay. 2005. The ants of North America. http://www3.utep.edu/leb/ antgenera.htm; última consulta: 24.I.2011.

Majer, D.J. 1983. Ants: Bio-indicators of minesite rehabilitation, land use, and land conservation. Environmental Management 7(4):375-383.

Mas, J.F., A. Velázquez, J.R. Díaz-Gallegos, R. Mayorga-Saucedo, C. Alcántara, G. Bocco, R. Castro, T. Fernández y A. Pérez-Vega. 2004. Assessing land use/cover changes: a nationwide multidate spatial data base for Mexico. International Journal of Applied Earth Observation and Geoinformation 5(4):249-261.

Mattoni, R., Longcore T. y V. Novotny. 2000. Environmental auditing arthropod monitoring for fine-scale habitat analysis: a case study of the El Segundo sand dunes. Environmental Management 25(4):445-452. 
Medina, J.G. y J.D. Tejero-Diez. 2006. Flora y vegetación del parque estatal Atizapán-Valle Escondido, Estado de México, México. Polibotánica 21:1-43.

Minchin, P.R. 1987. An evaluation of the relative robustness of techniques for ecological ordinations. Vegetatio 69(13):89-107.

Naeem, S., F.S. Chapin III, R. Constanza, P.R. Ehrlich, F.B. Golley, D.U. Hooper, J.H. Lawton, R.V. O’Neill, H.A. Mooney, O.E. Sala, A.J. Symstad y D. Tilman.1999. Biodiversity and ecosystem functioning: Maintaining natural life support processes. Issues in Ecology 4:1-11.

Philpott, S.M. y I. Armbrecht. 2006. Biodiversity in tropical agroforests and ecological role of ants and ant diversity in predatory function. Ecological Entomology 31(4):369-377.

Philpott, S.M., I. Perfecto, I. Armbrecht y C.L. Parr. 2010. Ant diversity and function in disturbed and changing habitats. In: L. Lach, C.L. Parr y K.L. Abbott, eds. Ant ecology. Oxford University Press. Oxford. p:137-156.

Premauer, J. y O. Vargas. 2004. Patrones de diversidad en vegetación pastoreada y quemada en un páramo húmedo (parque natural Chingaza, Colombia). Ecotropicos 17(1-2):52-66.

Rojas, P. 2001. Las hormigas del suelo en México: Diversidad, distribución e importancia (Hymenoptera: Formicidae). Acta Zoológica Mexicana (n. s.) número especial 1:189-238.

Rueppell, O. y R.W. Kirkman. 2005. Extraordinary starvation resistance in Temnothorax rugatulus (Hymenoptera, Formicidae) colonies: demography and adaptative behavior. Insectes Sociaux 52(3):282-290.

Rzedowski, J. 1992. Diversidad y orígenes de la flora fanerogámica de México. Ciencias número especial 6:47-56.

Schuh, R.T. y J.A. Slater. 1995. True bugs of the world (Hemiptera: Heteroptera) classification and natural history. Cornell University Press. Nueva York. 416 p.

Shah, P.A., D.R. Brooks, J.E. Ashby, J.N. Perry e I.P. Woiwod. 2003. Diversity and abundance of the coleopteran fauna from organic and conventional management systems in southern England. Agricultural and Forest Entomology 5(1):51-60.
Siemann, E., D. Tilman, J. Haarstad y M. Ritchie. 1998. Experimental test of the dependence of arthropod diversity on plant diversity. The American Naturalist 152(5):738750.

Sokal R.R. y J.F. Rohlf. 1995. Biometry. The principles and practice of statistic in biological research. 3a ed. W.H. Freeman and Company. Nueva York. 887 p.

Sonthichai, S., N. Gavinjan, S. Suwannaratana y W. Jaitrong. 2006. A comparison of ant populations in restored forest of different ages and adjacent natural vegetation in northern Thailand. Kasetsart Journal: Natural Science 40(4):882-889.

Stephens, S.S. y M.R. Wagner. 2006. Using ground foraging ant (Hymenoptera: Formicidae) functional group as bioindicators of forest health in northern Arizona ponderosa pine forest. Envrionmental Entomology 35(4):937-949.

Valencia A., S. 2004. Diversidad del género Quercus (Fagaceae) en México. Boletín de la Sociedad Botánica de México 75:33-53.

Whitford, E.G. 1978. Foraging in seed harvester-ants Pogonomyrmex spp. Ecology 59(1):185-189.

Wilby, A., M. Shachak. 2000. Harvester ant response to spatial and temporal heterogeneity in seed availability: pattern in the process of granivory. Oecologia 125(4):495-503.

Zavala, C.F. 1998. Observaciones sobre la distribución de encinos en México. Polibotánica 8:47-64.

Zavala-Hurtado, J.A., P.L. Valverde, M.C. Herrera-Fuentes y A. Díaz-Solís. 2000. Influence of leaf-cutting ants (Atta mexicana) on performance and dispersion patterns of perennial desert shrubs in an Inter-tropical region of Central Mexico. Journal of Arid Environments 46(1):93-102.

Manuscrito recibido el 14 de diciembre de 2011. Aceptado el 16 de agosto de 2013.

Este documento se debe citar como:

Guzmán-Mendoza, R., J.A. Zavala-Hurtado, G. Castaño-Meneses y J.L. León-Cortés. 2014. Comparación de la mirmecofauna en un gradiente de reforestación en bosques templados del centro occidente de México. Madera y Bosques 20(1):71-83. 